\title{
ON THE EXISTENCE OF AN INTEGER SOLUTION OF THE RELAXED WEBER PROBLEM FOR A TREE NETWORK
}

\author{
A.V. Panyukov, South Ural State University, Chelyabinsk, Russian Federation, \\ paniukovav@susu.ru
}

The problem of finding the optimal arrangement of vertices of a tree network in the installation space representing a finite set is considered. The criterion of optimality is the minimization of the total cost of deployment and the cost of communications. Placement of different tree vertices in one point of the installation space is allowed. This problem is known as Weber problem for a tree network. The statement of Weber problem as an integer linear programming problem is given in this research. It's proved that a set of optimal solutions of corresponding relaxed Weber problem for a tree-network contains the integer solution. This fact allows to prove the existence a saddle point while proving the performance of decomposition algorithms for problems different from problems because of additional restrictions.

Keywords: allocation problem; linear programming; duality; relaxation; integer solution; polynomial algorithm; Weber problem.

\section{Introduction}

The considered problem deals with finding the optimal arrangement of vertices of a tree network in the installation space representing a finite set $[1,2]$. The criterion of optimality is the minimization of the total deployment cost and of communication cost. Placement of different tree vertices in one point of the installation space is allowed. This problem is known as Weber problem for a tree network [3,4].

So, let's consider extremal problem $\Theta(G, V, b, c, \Phi)$

$$
C(\varphi)=\sum_{j \in J} c(j, \varphi(j))+\sum_{[i, j] \in E} b([i, j], \varphi(i), \varphi(j)) \rightarrow \min _{\varphi \in \Phi}
$$

for given tree $G=(J, E)$, finite set $V$, mapping $b: E \times V^{2} \rightarrow \mathbf{Z}^{\geq 0}$, mapping $c: J \times V \rightarrow$ $\mathbf{Z}^{\geq 0}$ and set $\Phi$ of allowed placements of set $J$ elements at points of set $V$.

If $\Phi=\{\varphi: J \rightarrow V\}$ (i.e. presents unambiguous maps) then problem $\Theta$ is known as Weber problem for a tree-connecting network. To solve this problem the polynomial algorithm [5] of complexity $O\left(|J||V|^{2}\right)$ is used.

The statement of Weber problem as an integer linear programming (ILP) problem is given in this paper. It's proved that a set of optimal solutions of corresponding relaxed Weber problem for a tree-network contains the integer solution. This fact allows to prove the existence of a saddle point while proving the performance of decomposition algorithms for problems different from a problem $\Theta$ because of additional restrictions $[1,6]$.

\section{Algorithm of Solving Weber Problem for a Tree Network}

To introduce the notation and simplify the presentation of main paper results, we give the algorithm for Weber problem from the author's work [5]. 
Let $N$ be a cardinality of set $J$, i.e. $N=|J|, j_{N} \in J$ be the end-vertex of tree $(J, E)$. The choice of vertex $j_{N}$ as a root of tree $(J, E)$ induces partial order

$$
P=\left\{(i, j): i, j \in J, j \text { belongs to a trail in }(J, E) \text { between } i \text { and } j_{N}\right\}
$$
of set $J$.

Let's assume that $J=\{k\}_{k=1}^{N}$ and corresponds condition $(l, m) \in P \Rightarrow l<m$. Let's designate the direct ancestor of vertex $l$ as $F(l)$, i.e. $F(l)=m:[l, m] \in E, l<m$. Pseudo-code of Weber problem $\Theta_{W}$ solution algorithm is presented in Fig. 1.

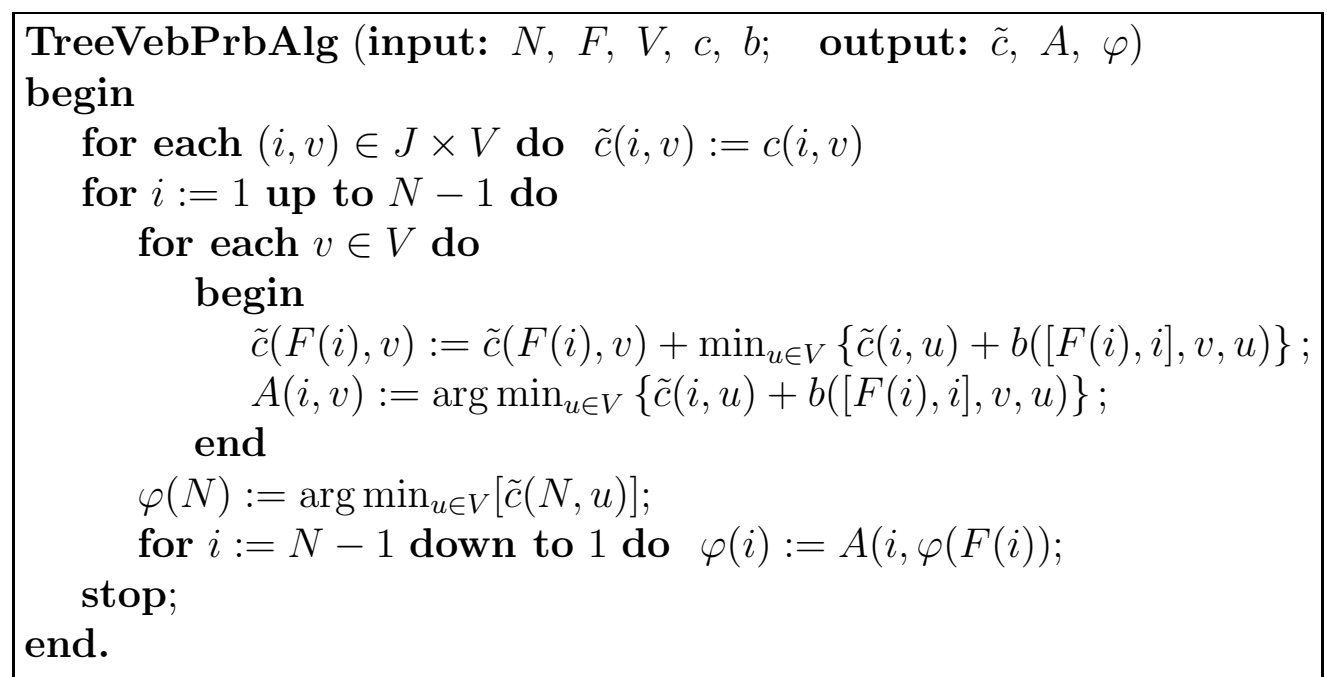

Fig. 1. Algorithm of Weber problem solution

The first stage of the algorithm means the sequential computation of $\forall v \in V$ pseudo-costs

$$
\tilde{c}(i, v):=c(i, v)+\sum_{j: i=F(j)} \min _{u \in V}[\tilde{c}(j, u)+b([i, j], v, u)], \quad i=1,2, \ldots, N
$$

and pseudo-placements

$$
A(i, v)=\arg \min _{u \in V}[\tilde{c}(i, u)+b([F(i), i], u, v)], \quad i=1,2, \ldots, N
$$

and the second stage is the sequential computing of optimal placement for tree vertices

$$
\varphi(N):=\arg \min _{u \in V}[\tilde{c}(N, u)], \quad \varphi(j)=A(j, \varphi(F(j))), j=N-1, \ldots, 1 .
$$

The optimal solution of Weber problem is equal to $\min _{u \in V}[\tilde{c}(N, u)]$. The computing complexity of algorithm TreeVebPrbAlg is $O\left(|J||V|^{2}\right)$. For more details and proofs see [5].

\section{Relaxed Weber Problem}

Let's consider integer linear programming problem

$$
C(y, z)=\sum_{j \in J, v \in V} y_{v}^{j} c(j, v)+\sum_{[i, j] \in E, v, u \in V} z_{v u}^{i j} b([i, j], v, u) \rightarrow \min _{(y, z) \in M}
$$

allowed set $M$ of which is defined by a system of restrictions

$$
\sum_{v \in V} y_{v}^{i}=1, i \in J
$$




$$
\begin{gathered}
\sum_{u \in V} z_{v u}^{i j}=y_{v}^{i}, \sum_{u \in V} z_{u v}^{i j}=y_{v}^{j},[i, j] \in E, v \in V ; \\
y \geq 0, \quad z \geq 0 \\
y_{v}^{i} \in\{0,1\}, i \in J, v \in V ; \\
z_{v u}^{i j} \in\{0,1\}[i, j] \in E, v, u \in V .
\end{gathered}
$$

Let's show that there exists 1-1-correspondence between the set of allowed solutions for problem (5) - (10) and problem $\Theta$ saving the value of a goal function.

Let $\varphi \in \Phi$. Define $(y, z)$ as following

$$
\begin{array}{cl}
y_{v}^{i}=\chi_{\{v\}}(\varphi(i)), & i \in J, v \in V, \\
z_{v u}^{i j}=\chi_{\{(v, u)\}}(\varphi(i), \varphi(j)), & {[i, j] \in E, v, u \in V,}
\end{array}
$$

where $\chi_{X}(\cdot)$ be a characteristic function of set $X$. The equality $C(\varphi)=C(y, z)$ is obvious in this case. Inclusion $(y, z) \in M$ follows from uniqueness of mapping $\varphi$.

Conversely if $(y, z) \in M$ then according to (6) and (9) function $\varphi: J \rightarrow V: \varphi(i)=$ $v: y_{v}^{i}=1$ be unique. Moreover, (7) and (10) imply $z_{v u}^{i j}=1$ if and only if $y_{v}^{i}=y_{u}^{j}=1$. Hence, equality $C(\varphi)=C(y, z)$ takes place.

So, it's possible to formulate $\Theta$ as a problem of integer linear programming $(5)-(10)$. Later we designate problem (5) - (10) also as Weber problem $\Theta$, and functions $\varphi: J \rightarrow V$ be its solutions, variables $(y, z)$ are determined according to $(11)-(12)$.

Let's call linear programming problem (5) - (8) varying of Weber problem due to absence of integrality conditions $(9)-(10)$ as relaxed Weber problem $\tilde{\Theta}$. Let's designate the allowed set for this problem as $\tilde{M}$. Some properties of polyhedron for relaxed Weber problem are investigated in [7]. The key result of the current paper is announced in [8].

Theorem 1. If $G$ is a tree then the optimal solution of Weber problem $\Theta$ is also the optimal solution of corresponding relaxed Weber problem $\tilde{\Theta}$.

Proof. Problem $\tilde{\Theta}^{*}$

$$
\tilde{C}^{*}(x, w)=\sum_{j \in J} x_{j} \rightarrow \max _{(x, w) \in \tilde{M}^{*}}
$$

with allowed set $\tilde{M}^{*}$ defined by relations

$$
\begin{aligned}
& x_{i}-\sum_{j: i=F(j)} w_{v *}^{i j}-w_{*}^{F(i) i} \leq c(i, v), \quad(i, v) \in J \times V, \\
& \left.w_{v *}^{i j}+w_{* u}^{i j} \leq b([i, j], v, u), \quad([i, j], v, u) \in E \times V^{2}\right)
\end{aligned}
$$

is dual to relaxed Weber problem $\tilde{\Theta}$.

To prove the theorem it's enough to construct the allowed solution of $(13)-(15)$ satisfying the complementary slackness conditions on the decision solution $\varphi \in \Phi$ of problem $\Theta(G, V, b, c, \Phi)$ constructed using the algorithm TreeVebPrbAlg.

Let

$$
w_{v}^{F(i) i}=\min _{u \in V}[\tilde{c}(i, u)+b([F(i), i], v, u)], \quad(i, v) \in J \times V .
$$


It follows from (4) and (16) that

$$
w_{\varphi(F(i)) *}^{F(i)}=\tilde{c}(i, \varphi(i))+b([F(i), i], \varphi(F(i)), \varphi(i)) .
$$

Because the optimal solution has $z_{\varphi(i) \varphi(j)}^{i}=1$ then corresponding restrictions (15) should be active. It allows to define

$$
w_{*}^{F(i) i}{ }_{\varphi(i)}=b([F(i), i], \varphi(F(i)), \varphi(i))-w_{\varphi(F(i)) *}^{F(i)} \quad i=-\tilde{c}(i, \varphi(i)), \quad i \in J .
$$

Assuming

$$
w_{*}^{F(i) i}{ }_{v}=\min _{u \in V}\left\{b([F(i), i], u, v)-w_{u}^{F(i) i}{ }_{*}\right\}, \quad i \in J, v \in V \backslash\{\varphi(j)\},
$$

we get vector $w$ satisfying all restrictions (15).

The condition of activeness of restrictions for corresponding variables $y_{\varphi(i)}^{i}$ allows to define

$$
\begin{aligned}
& x_{i}=c(i, \varphi(i))+\sum_{j: i=F(j)} w_{\varphi(i) *}^{i}+w_{*}^{F(i) i} \varphi(i)=\tilde{c}(i, \varphi(i))+w_{*}^{F(i) i} \varphi(i)= \\
& \quad= \begin{cases}\tilde{c}(N, \varphi(N)), & i=N, \\
0, & i \in J \backslash\{N\} .\end{cases}
\end{aligned}
$$

The second equality in this circuit is a consequence of (3) and (17) and the last one is a consequence of (18) and abcence of ancestor of vertex $N$.

For all other values of $v \in V \backslash \varphi(J)$ we have

$$
\begin{gathered}
c(i, v)+\sum_{j: i=F(j)} w_{v *}^{i j}+w_{*}^{F(i) i}=\tilde{c}(i, v)+w_{*}^{F(i) i}=\tilde{c}(i, v)+\min _{u \in V}\left\{b([F(i), i], u, v)-w_{u}^{F(i) i}{ }_{*}\right\} \geq \\
\geq \min _{v \in V}\left\{\tilde{c}(i, v)+b\left([F(i), i], l^{*}, v\right)\right\}-w_{l^{*}}^{F(i) i}{ }_{*}^{(i)}=0
\end{gathered}
$$

for restrictions system (14). The first equality in circuit (21) is a consequence of (3) and (17), the second one is a consequence of (19). The inequality is obvious for

$$
l^{*}=\arg \min _{l \in V}\left(b([i, F(i)], v, l)-w_{l}^{F(i) i} *\right)
$$

and the last equality follows from (16).

That's why the solution $(x, w)$ obtained according to (16) - (20) is allowed solution of problem (13) - (15) satisfying the conditions of complementary slackness relatively to solution $\varphi \in \Phi$ of problem $\Theta(G, V, b, c, \Phi)$ constructed by algorithm. Hence, it is the optimal solution of $(13)-(15)$. Theorem 1 now follows. At the same time, the way of defining the integer optimal solution for $(13)-(15)$ is shown.

\section{Conclusion}

So, it's proved that a set of optimal solutions of corresponding relaxed Weber problem for a tree-network contains the integer solution. This fact allows to prove the existence of a saddle point while proving the performance of decomposition algorithms for problems different from problem $\Theta_{V}$ because of additional restrictions. 


\section{References}

1. Beresnev V.L., Diskretnye zadachi razmeshcheniya $i$ polinomy ot bulevykh peremennykh [Discrete Location Problems and Polynomials of Boolean Variables]. Novosibirsk, Sobolev Institute Press, 2005. (in Russian)

2. Nickel S., Puerto J. Location Theory. Heidelberg, Springer, 2005.

3. Zabudskii G.G., Veremchuk N.S. An Algorithm for Finding an Approximate Solution to the Weber Problem on a Line with Forbidden Gaps. Journal of Applied and Industrial Mathematics, 2016, vol. 10, no. 1, pp. 136-144. DOI: 10.1134/S1990478916010154

4. Zabudskii G.G., Koval A.A. Solving a Maximin Location Problem on the Plane with Given Accuracy. Automation and Remote Control, 2014, vol. 75, pp. 1221-1230. DOI:10.1134/S0005117914070042

5. Panyukov A.V., Pelzwerger B.V. Polynomial Algorithms to Finite Weber Problem for a Tree Network. Journal of Computational and Applied Mathematics, 1991, vol. 35, pp. 291-296. DOI:10.1016/0377-0427(91)90215-6

6. Ivanko E.E. Iterative Equitable Partition of Graph as a Model of Constant Structure Discrete Time Closed Semantic System. Bulletin of the South Ural State University. Series: Mathematical Modelling, Programming and Computer Software, 2017, vol. 10, no. 4, pp. 26-34. DOI: 10.14529/mmp170403

7. Panyukov A.V. The Relaxation Polyhedron of Weber Problem. Non-Smooth and Discontinuous Problems of Control and Optimization, Chelyabinsk, 1998, pp. 171-174.

8. Panyukov A.V. Location of a Tree Network for a Finite Set. Abstracts of the Seventh Czech-Slovak International Symposium on Graph Theory, Combinatorics, Algorithms and Applications, Kosice, Safary University, 2013, p. 64.

\section{О СУЩЕСТВОВАНИИ ЦЕЛОЧИСЛЕННОГО РЕШЕНИЯ РЕЛАКСИРОВАННОЙ ЗАДАЧИ ВЕБЕРА ДЛЯ ДРЕВОВИДНОЙ СЕТИ}

A.B. Панюков, Южно-Уральский государственный университет, г. Челябинск, Российская Федерация

Рассмотрена задача нахождения оптимального размещения вершин древовидной сети в монтажном пространстве, представляющем конечное множество. Критерием оптимальности является минимизация общей стоимости размещения в точках пространства и стоимости коммуникаций. Допускается размещение разных вершин дерева в одной точке монтажного пространства. Рассматриваемая проблема известна как задача Вебера для древовидной сети. В данной работе дано представление задачи Вебера как задачи о линейном программировании. Доказано, что множество оптимальных решений соответствующей релаксированной задачи Вебера для древовидной сети содержит целочисленное решение. Этот факт позволяет доказать существование седловой точки 
при доказательстве эффективности алгоритмов декомпозиции для задач, отличающихся от задачи Вебера наличием дополнительных ограничений.

Ключевые слова: задача размещения; линейное программирование; двойственность; релаксация; целочисленное решение; полиномиальный алгоритм; задача Вебера.

Анатолий Васильевич Панюков, доктор физико-математических наук, профессор, кафедра «Математическое и компьютерное моделирование», Южно-Уральский государственный университет (г. Челябинск, Российская Федерация), paniukovav@susu.ru.

Поступила в редакиию 3 августа 2018 г. 